\title{
Erythrocyte phospholipid molecular species and fatty acids of Down syndrome children compared with non-affected siblings
}

\author{
Allain A. Bueno ${ }^{1}$, Annette Brand ${ }^{2}$, Marita M. Neville ${ }^{3}$, Catherine Lehane ${ }^{3}$, Nina Brierley ${ }^{4}$ and \\ Michael A. Crawford ${ }^{5 *}$ \\ ${ }^{1}$ Institute of Science and the Environment, University of Worcester, Henwick Grove, Worcester WR2 6AJ, UK \\ ${ }^{2}$ Department of Neurobiology, The Weizmann Institute of Science, Rebovot 76100, Israel \\ ${ }^{3}$ Institute of Brain Chemistry and Human Nutrition, London Metropolitan University, 166-220 Holloway Road, \\ London $N 7$ 8DB, UK \\ ${ }^{4}$ The Harley Street Clinic, 35 Weymouth Street, London W1G 8BJ, UK \\ ${ }^{5}$ Reproductive Physiology, Imperial College, London, Chelsea and Westminster Hospital Campus, 369 Fulham Road, \\ London SW1O 9NH, UK \\ (Submitted 16 June 2014 - Final revision received 25 August 2014 - Accepted 15 September 2014 - First published online 24 November 2014)
}

\begin{abstract}
The majority of children with Down syndrome (DS) develop Alzheimer's disease (AD) at an early age. Although long-chain $n$ - 3 fatty acids (FA) are protective of neurodegeneration, little is known about the FA status in DS. In the present study, we aimed to investigate whether children with DS presented altered plasma and erythrocyte membrane phospholipids (PL) FA composition, when compared with their nonaffected siblings. Venous blood samples were analysed for plasma and erythrocyte membrane FA composition by TLC followed by GC techniques. Lipid molecular species were determined by electrospray ionisation/tandem MS (ESI-MS/MS). FA analysis measured by standard GC showed an increased concentration of MUFA and a decreased concentration of plasmalogens in major PL fractions, but there were no differences in the concentrations of arachidonic acid or DHA. However, as identified by ESI-MS/MS, children with DS had increased levels of the following erythrocyte PL molecular species: 16:0-16:0, 16:0-18:1 and 16:0-18:2n-6, with reduced levels of 16:0-20:4n-6 species. Children with DS presented significantly higher levels of MUFA in both plasma and erythrocyte membrane, as well as higher levels of saturated and monounsaturated molecular species. Of interest was the almost double proportion of $16: 0-18: 2 n-6$ and nearly half the proportion of 16:0-20:4n-6 of choline phosphoacylglycerol species in children with DS compared with their non-affected siblings. These significant differences were only revealed by ESI-MS/MS and were not observed in the GC analysis. Further investigations are needed to explore molecular mechanisms and to test the association between the pathophysiology of DS and the risk of $\mathrm{AD}$.
\end{abstract}

Key words: Down syndrome: Phospholipids: MS: Alzheimer's disease

Down syndrome (DS) is a major genetic disorder caused by trisomy of chromosome 21, leading to recognisable physical and facial features and several chronic disorders. The prevalence of this disorder is approximately one in 660 live births $^{(1)}$, and it increases with maternal age ${ }^{(2)}$. DS is the leading cause of genetically defined intellectual disability and congenital birth defects.

In the UK, one to two children with DS are born every day, and they present a much higher risk of developing leukaemia, diabetes, heart disease, premature ageing and dementia, and Alzheimer's disease (AD)-like pathology at the age of $30-40$ years $^{(3,4)}$ when compared with non-affected children.
Early onset of AD has been causally related to the overexpression of $\beta$-amyloid precursor protein, which is one of the triple genes in $\mathrm{DS}^{(5,6)}$.

Another gene located on chromosome 21 is the superoxide dismutase ( $S O D-1)$ gene. Its overexpression leads to a dramatic increase in SOD-1 enzyme activity ${ }^{(7,8)}$. This consequence is thought to disturb the natural antioxidant enzyme balance in individuals with DS, inducing oxidative stress ${ }^{(9,10)}$. Increased levels of oxidative stress have been detected in children with $\mathrm{DS}^{(11)}$, and concomitantly higher activities of other antioxidant enzymes, including glutathione peroxidase (GPx) and catalase, have also been reported to be increased ${ }^{(12,13)}$.

Abbreviations: AD, Alzheimer's disease; CerPCho, sphingomyelin; ChoGpl, choline glycerophospholipids; DMA, fatty aldehyde dimethylacetals; DS, Down syndrome; ESI-MS/MS, electrospray ionisation/tandem MS; FA, fatty acids; GPx, glutathione peroxidase; HMBC, Holloway Mother and Baby Clinic; PL, phospholipids; PtdEtn, phosphatidylethanolamine; PtdSer, phosphatidylserine; SOD, superoxide dismutase.

*Corresponding author: Professor M. A. Crawford, fax +44 208846 7892, email michael.crawford@imperial.ac.uk 
The brain is particularly vulnerable to lipid peroxidation ${ }^{(14)}$ due to its high content of oxidisable PUFA, especially DHA (22:6n-3), which is a major plasma membrane fatty acid (FA) component, essential for brain development, signal transduction and synaptic function ${ }^{(15)}$. Increased lipid peroxidation has been suggested as a mechanism leading to $\mathrm{AD}^{(16)}$, and even more specifically, chronic losses of DHA are thought to contribute to $\mathrm{AD}^{(17,18)}$. Furthermore, low levels of EPA (20:5n-3), one of the precursors of DHA, are considered as a predictor of dementia risk ${ }^{(19)}$. Moreover, neuroprotectin D1, a derivative of DHA, has been reported to preserve neuronal systems from oxidative stress and potentially protect from neurodegeneration as in $\mathrm{AD}^{(20)}$, but only very little information is available about the status of PUFA in people with DS.

Cell membrane lipid composition is directly influenced by environmental variables, particularly diet, and abnormalities of membrane lipid composition and metabolism have been implicated in type 2 diabetes $^{(21)}$, obesity ${ }^{(22)}$ and $\mathrm{AD}^{(23)}$. It is not known yet whether the cell membrane FA composition is altered in children with DS, or whether any alteration in their cell membrane lipids might be related to the increased risk of developing diabetes, obesity, early mental decline and AD. Nutrition has been suggested as an interventional strategy aiming to prevent or reduce glycoxidation damage that appears to play a role in cognitive deterioration and the early onset of dementia in people with $\mathrm{DS}^{(24)}$; however, no data are available about the influence of PUFA supplements on the well-being of people with DS.

The aim of the present study was to investigate whether the extra copy of chromosome 21 has an influence on the FA composition of phospholipids (PL) in the erythrocyte membrane of children with DS, when compared with their non-affected siblings. TLC, GC and electrospray ionisation/ tandem MS (ESI-MS/MS) were employed to study the possible changes in the PUFA profile and intact PL molecular species. Our data provide evidence that the FA profile of PL in erythrocyte membrane and plasma is significantly modified in children with DS. While PUFA and plasmalogen species are markedly reduced, the content of SFA and MUFA is dramatically increased.

\section{Subjects and methods}

\section{Subjects}

A total of twenty-five families with children with DS and their non-affected siblings volunteered for the present study conducted at the Holloway Mother and Baby Clinic (HMBC), at London Metropolitan University, through advertisement posted on the Down Syndrome Research Foundation website. We aimed to compare children with DS with their unaffected siblings to help control for family backgrounds, dietary patterns and social status, which could interfere with the outcomes of the study. The present study was conducted according to the guidelines laid down in the Declaration of Helsinki, and all procedures were approved by the Ethics Committee of London Metropolitan University, and endorsed by the Down Syndrome Research Foundation. Written informed consent was obtained from the parents, and verbal consent was obtained from the children, witnessed and formally recorded.

\section{Diet analysis}

A $7 \mathrm{~d}$ food diary was obtained from all the participants before the interview, sent by post to the State Registered Dietitian, and analysed. The records were analysed by the dietitian using the software/database FOODBASE version 3.1, which is based on 'McCance and Widdowson's The Composition of Foods' and its ten accompanying supplements ${ }^{(25)}$, and enhanced FA data produced by the Institute of Brain Chemistry and Human Nutrition. The food diary and nutrition software has been validated previously ${ }^{(26)}$. The diet provided thorough data on nutrient intake and, particularly, each member of the $n-6$ and $n-3$ FA classes.

Subsequent to the food diary, the families were invited by the dietitians (N. B. and M. M. N.) for an interview at the HMBC. Dietetic advice was given to the families based on the diet analysis, and particular attention was paid to diet habits, details that might have been under- or over-reported in the diaries, and the use of nutrient supplements. Children who were taking vitamins or food supplements, even sporadically, were excluded from the present study, as well as children who were taking food supplements up to 2 months before the appointment. A number of children were consuming fish oil or other nutritional supplements; consequently, both the child with DS and the non-affected sibling were excluded. Only the results for nine families are reported in the present study.

\section{Blood collection}

A non-fasting $3 \mathrm{ml}$ venous blood sample was collected in EDTA tubes at the HMBC by a locum nurse after the interview. The blood sample was immediately taken to the Institute of Brain Chemistry and Human Nutrition laboratory, centrifuged, and plasma, buffy coat and erythrocyte fractions were obtained. Erythrocytes were washed three times with Dulbecco's PBS (Sigma). All aliquots were flushed with oxygen-free $\mathrm{N}_{2}$ and frozen at $-80^{\circ} \mathrm{C}$ until analysis.

\section{Lipid extraction and fatty acid analysis}

Total lipids were extracted from erythrocytes and plasma by the method of Folch et al. ${ }^{(27)}$, and FA analysis was performed according to standard procedures as reported previously ${ }^{(28)}$. Briefly, erythrocyte samples $(1 \mathrm{ml})$ were homogenised in $45 \mathrm{ml}$ chloroform-methanol $(2: 1, \mathrm{v} / \mathrm{v})$ containing $0 \cdot 01 \%$ butylated hydroxytoluene under oxygen-free $\mathrm{N}_{2}$. Ethanolamine, choline, serine phosphoacylglycerols (PtdEtn, ChoGpl and PtdSer) and sphingomyelin (CerPCho) were separated with chloroform-methanol-methylamine (65:35:15, by vol.) on $20 \times 20 \mathrm{~cm}^{2}$ silica gel TLC plates (VWR International) at room temperature. Plasma samples were extracted and separated in the same manner. The plasma neutral lipids 
cholesterol esters and TAG were scraped from the plasma TLC plates, washed with petroleum ether-diethyl ether (50:50, $\mathrm{v} / \mathrm{v})$ and separated with petroleum ether-diethyl etherformic acid-methanol ( $85: 15: 2 \cdot 5: 1$, by vol.) on TLC plates at room temperature. The lipid bands were detected by spraying with 2,7-dichlorofluorescein $(0.01 \%, \mathrm{w} / \mathrm{v})$ in methanol, visualised under UV light and compared with authentic standards (Sigma). FA methyl esters were obtained by heating the PL fractions scraped from the TLC plates with $15 \%$ acetyl chloride in dry methanol in a sealed tube at $70^{\circ} \mathrm{C}$ for $3 \mathrm{~h}$ under oxygen-free $\mathrm{N}_{2}$. The reaction was stopped by the addition of $5 \% \mathrm{NaCl}$ solution at room temperature, and FA methyl ester were extracted by washing three times with petroleum ether containing $0.01 \%$ butylated hydroxytoluene. FA methyl ester were separated by GC (HRGC MEGA 2 series; Fisons Instruments) fitted with a capillary column (BPX70; Thames Restek). Peak areas were quantified (EZChrom Chromatography Data System; Scientific Software, Inc.), values are expressed as the percentage of total FA, and the calculation of area percentage was assumed to be equivalent to weight percentage.

\section{Enzyme activity determination}

Erythrocyte SOD $(\mathrm{U} / \mathrm{ml}$; where one unit is defined as the amount of enzyme needed to exhibit $50 \%$ dismutation of the superoxide radical), GPx ( $\mathrm{nmol} / \mathrm{min}$ per $\mathrm{ml}$ ) and catalase ( $\mathrm{nmol} / \mathrm{min}$ per $\mathrm{ml}$ ) were analysed by commercially available ELISA kits (Cayman Chemicals), according to the instructions of the manufacturer.

\section{Electrospray ionisation-MS/MS}

ESI-MS/MS measurements were performed on a Q-TOF Micro hybrid quadrupole time-of-flight mass spectrometer (Waters Corporation), as described previously ${ }^{(29)}$. Briefly, erythrocyte lipids were extracted with a mixture of hexane-isopropanol $(3: 2, \mathrm{v} / \mathrm{v})$ containing $0 \cdot 01 \%$ butylated hydroxytoluene. The samples were washed with chloroform-methanol-water (2:1:1, by vol.). After low-speed centrifugation, the organic layer was isolated, evaporated to dryness and lipids partitioned again in the chloroform layer of a chloroformmethanol-water solution (8:4:3, by vol.). The chloroform layer containing the crude lipid extracts was evaporated to dryness and dissolved in methanol-chloroform $(2: 1, \mathrm{v} / \mathrm{v})$ to a concentration $<5 \mathrm{pmol} / \mathrm{ml}$ and infused directly into the ESI chamber at a flow rate of $5-8 \mu \mathrm{l} / \mathrm{min}$. Typically, a $1-2 \mathrm{~min}$ period of signal averaging was employed for each spectrum. The capillary was operated at $-2.5 \mathrm{kV}$ in the positive-ion mode. Desolvation temperature was maintained at $180^{\circ} \mathrm{C}$. MS data were processed with MassLynx 4.0 software (Waters Corporation) to produce peak list files. The identities of all indicated molecular species were confirmed by ESI-MS/ MS. To acquire suitable product-ion spectra, collision energies were varied between 25 and $35 \mathrm{eV} .{ }^{13} \mathrm{C}$ isotope effects were corrected by determining the individual type II ${ }^{13} \mathrm{C}$ isotope correction factor, as described previously ${ }^{(30)}$. For positive-ion analyses and identification of positively charged PL species, aliquots of lipid extracts were treated with $\mathrm{CHCl}_{3}-\mathrm{CH}_{3} \mathrm{OH}-$

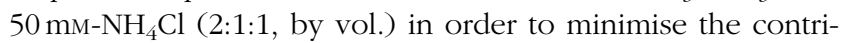
butions of metal ions allowing the formation of $[\mathrm{M}+\mathrm{H}]^{+}$ adducts, thus simplifying ESI-MS/MS spectra. Subsequently, $\mathrm{LiOH}$ (final concentration $2 \mathrm{~mm}$ ) was added to the samples to allow the formation of $[\mathrm{M}+\mathrm{Li}]^{+}$adducts and facilitate informative fragmentation by MS/MS. These samples were not used for quantification as alkaline treatment might induce some PL hydrolysis.

\section{Statistical analysis}

Results are presented as means and standard deviations. For the analysis of PL molecular species, we tested for differences between children with DS and their non-affected siblings by the Mann-Whitney $U$ test. For the other analysis, Student's $t$ test was used. Differences were considered statistically significant at $P<0 \cdot 05$. Details about sample sizes are given in the individual table and figure legends.

\section{Results}

A total of twenty-five families with children with DS and their non-affected siblings volunteered for the present study; however, not all children were able to participate fully in the study. The analysis of a $7 \mathrm{~d}$ food diary for each child revealed significant differences in their $n-9, n-6$ and $n-3$ FA intake due to PUFA-containing food supplements in various concentrations, from various suppliers. Hence, children who were taking any fish oils, vitamins or other supplements, even sporadically, were excluded from the present study, as well as those who had taken supplements, up to 2 months before the appointment and blood sampling. Thus, only children from nine families were included in the present study. The average age for healthy siblings was $9 \cdot 2$ (SD 4.4) years, and for children with DS was 5.9 (SD 3.7) years (statistically similar). The study contained four boys and seven girls in the healthy sibling group, and five boys and four girls in the DS group (Table 1). The average intakes of energy and nutrients, specifically $n-3, n-6$ and $n$-9 FA, were similar between children with DS and their unaffected siblings (data not shown).

\section{Lipid analysis}

Blood samples were collected and lipid extracts were prepared from erythrocytes and plasma. Following separation of the various PL fractions by TLC, GC analysis revealed the

Table 1. Age and sex of children with Down syndrome (DS) and their healthy siblings (HS)

(Mean values with standard deviations, $n 11$ for $\mathrm{HS}$ and $n 9$ for DS)

\begin{tabular}{lccccccc}
\hline & \multicolumn{3}{c}{ HS } & & \multicolumn{2}{c}{ DS } \\
\cline { 2 - 4 } & Mean & & SD & & Mean & SD \\
\hline Age (years) & 9.2 & & 4.4 & & 5.9 & & 3.7 \\
Boys $(n)$ & & 4 & & & 5 & \\
Girls $(n)$ & & 7 & & & 4 & \\
\hline
\end{tabular}


changes in the FA profile of PL fractions in erythrocyte membrane, as well as changes in PL and neutral lipid fractions in plasma. The major PL classes, i.e. the aminophosphoacylglycerols (PtdEtn and PtdSer) which are rich in PUFA, and the rather SFA-rich PL (ChoGpl and CerPCho) of erythrocyte membrane were analysed, as well as plasma ChoGpl, TAG and $\mathrm{CE}$.

In the erythrocyte membrane PtdEtn of children with DS, the amount of the major SFA 16:0 was increased by $14 \%$, causing the total amount of SFA to be elevated when compared with the samples of healthy siblings (Table 2). The amount of the major MUFA $18: 1 n-9$ was increased by $7 \cdot 3 \%$, and that of a minor MUFA, $18: 1 n-7$, was elevated by $16 \%$. In contrast to the other PL classes, PtdEtn is rather rich in a particular group of phosphoacylglycerols measurable by GC analysis as fatty aldehyde dimethylacetals (DMA). DMA species are the cleavage products of 1-alkenyl 2-acyl phosphoacylglycerol species, also known as plasmalogens. Plasmalogens consist of an aldehyde in the $s n$ - 1 position, giving rise to a single vinyl ether double bond and a long-chain hydrocarbon containing a carboxyl group esterified to the $s n-2$ position of the glycerol moiety possessing between two to six double bonds. The amount of 18:0 DMA, reflecting the major plasmalogen PtdEtn species in erythrocyte membrane, was reduced by more than $20 \%$ in children with DS.

Of the $n$ - 6 PUFA families, the amounts of two $n-6$ FA (18:2n-6 and $22: 4 n-6$ ) were found to be altered in the erythrocyte membrane PtdEtn of children with DS. The amount of dietary essential FA linoleic acid (18:2n-6) was increased by $15 \%$, while its metabolic elongation and desaturation product $22: 4 n-6$ was decreased by $18 \%$. Consequently, the ratio $18: 2 n-6 / 20: 4 n-6$ was increased and the ratio $22: 4 n-6 / 22: 5 n-6$ was decreased. There were no statistically significant changes in the distribution of $n-3$ PUFA (Table 2) in this PL fraction.

Analysis of the FA profile in erythrocyte membrane PtdSer, the second PUFA-rich PL class, revealed no significant differences in the amounts of SFA, MUFA or $n-3$ and n-6 PUFA. However, the amount of 18:0 DMA, a minor species in PtdSer since plasmalogens are not as prominent in PtdSer as in PtdEtn, was reduced on average by $68 \%$ in children with DS compared with their healthy siblings.

ChoGpl represents the major PL class in erythrocyte membrane as well as in plasma. In the erythrocyte membrane ChoGpl of children with DS, the amount of the major SFA 16:0 was increased from 33 to $36 \%$ of the total ChoGpl FA, while that of 18:0 was decreased from 12 to $11 \%$, resulting in an elevated amount of SFA when compared with the samples of unaffected siblings (Table 3). A similar trend could be found in plasma ChoGpl, although the numbers were less significant (Table 3). While the major MUFA (18:1n-9) in erythrocyte membrane and plasma ChoGpl remained unchanged, its isomer $18: 1 n-7$ was increased from 1.5 to $1.8 \%$ (a $20 \%$ increase), and from 1.3 to $1.7 \%$ (a $31 \%$ increase), in erythrocyte membrane and plasma ChoGpl, respectively. In addition, another $n-7 \mathrm{FA}$, palmitoleic acid (16:1n-7), was significantly elevated in plasma ChoGpl.

Table 2. Fatty acid (FA) composition of erythrocyte ethanolamine (PtdEtn) and serine phosphoacylglycerols (PtdSer) in children with Down syndrome (DS) when compared with their healthy siblings (HS)

(Mean values and standard deviations of total FA (\%), $n 11$ for HS and $n 9$ for DS)

\begin{tabular}{|c|c|c|c|c|c|c|c|c|}
\hline & \multicolumn{4}{|c|}{ PtdEtn } & \multicolumn{4}{|c|}{ PtdSer } \\
\hline & \multicolumn{2}{|c|}{$\mathrm{HS}$} & \multicolumn{2}{|c|}{ DS } & \multicolumn{2}{|c|}{$\mathrm{HS}$} & \multicolumn{2}{|c|}{ DS } \\
\hline & Mean & SD & Mean & SD & Mean & SD & Mean & SD \\
\hline $16: 0$ & $12 \cdot 85$ & 0.87 & $14 \cdot 69^{\star \star \star}$ & 0.75 & 5.73 & 1.79 & $5 \cdot 28$ & 1.77 \\
\hline $18: 0$ & 5.68 & 0.52 & 5.84 & 0.60 & 39.53 & $2 \cdot 64$ & 40.79 & 1.95 \\
\hline ¿SFA & 18.91 & $1 \cdot 12$ & $21.07^{\star \star \star}$ & 1.28 & $46 \cdot 19$ & 2.97 & 46.92 & 1.78 \\
\hline $16: 1 n-7$ & 0.19 & 0.04 & 0.20 & $0 \cdot 10$ & 0.27 & 0.55 & 0.12 & 0.07 \\
\hline $18: 1 n-9$ & $14 \cdot 76$ & 1.02 & $15 \cdot 84^{*}$ & 1.31 & $7 \cdot 88$ & $2 \cdot 30$ & $6 \cdot 41$ & 1.08 \\
\hline $18: 1 n-7$ & 0.87 & 0.12 & $1 \cdot 01^{*}$ & 0.10 & 0.66 & 0.16 & 0.77 & 0.23 \\
\hline$\Sigma M U F A$ & $16 \cdot 25$ & $1 \cdot 10$ & $17 \cdot 55^{\star}$ & 1.47 & 9.56 & $3 \cdot 15$ & 7.94 & 1.38 \\
\hline$\Sigma n-7$ & 1.05 & 0.15 & $1 \cdot 21^{*}$ & 0.15 & 0.93 & 0.59 & 0.89 & 0.26 \\
\hline $18: 0 / 18: 1$ & 0.37 & 0.05 & 0.35 & 0.04 & 4.98 & 1.40 & 5.92 & 1.64 \\
\hline $16: 0$ DMA & 4.79 & 0.56 & $4 \cdot 26$ & 0.76 & 0.18 & 0.11 & 0.14 & 0.11 \\
\hline 18:0 DMA & $9 \cdot 30$ & 0.84 & $7 \cdot 33^{\star \star \star}$ & 0.78 & 0.25 & 0.09 & $0.17^{*}$ & 0.06 \\
\hline ¿DMA & $15 \cdot 57$ & 1.41 & $13 \cdot 02^{\star \star \star}$ & 1.60 & 0.57 & 0.33 & 0.35 & 0.16 \\
\hline $18: 2 n-6$ & $5 \cdot 38$ & 0.73 & $6 \cdot 19$ & 1.04 & 4.13 & 1.25 & 3.94 & 1.23 \\
\hline $20: 4 n-6$ & $22 \cdot 20$ & 1.58 & $20 \cdot 97$ & 1.90 & $23 \cdot 19$ & $4 \cdot 10$ & $22 \cdot 38$ & 3.87 \\
\hline $22: 4 n-6$ & 5.83 & 1.04 & $4.80^{*}$ & 0.72 & $2 \cdot 30$ & 0.52 & 2.55 & 0.49 \\
\hline$\sum n-6$ PUFA & 35.92 & $2 \cdot 35$ & 34.46 & $2 \cdot 66$ & $33 \cdot 24$ & 4.65 & $32 \cdot 68$ & 2.99 \\
\hline $18: 2 n-6 / 20: 4 n-6$ & 0.24 & 0.03 & $0.30^{*}$ & 0.06 & 0.19 & 0.07 & 0.18 & 0.08 \\
\hline $22: 4 n-6 / 22: 5 n-6$ & $7 \cdot 23$ & 0.82 & $5 \cdot 89^{\star *}$ & 0.78 & 2.41 & 0.41 & $2 \cdot 46$ & 0.77 \\
\hline $20: 5 n-3$ & 1.59 & 0.77 & 1.45 & 0.58 & 0.58 & 0.28 & 0.49 & 0.16 \\
\hline $22: 6 n-3$ & 6.35 & 1.32 & 6.64 & $2 \cdot 27$ & 5.99 & 2.37 & $7 \cdot 23$ & 2.44 \\
\hline$\sum n-3$ PUFA & $12 \cdot 26$ & $2 \cdot 25$ & $12 \cdot 78$ & $2 \cdot 62$ & 9.47 & 2.95 & 11.68 & 2.58 \\
\hline
\end{tabular}

DMA, fatty aldehyde dimethylacetals.

Mean value was significantly different from that of the HS group: ${ }^{\star} P<0.05,{ }^{\star \star} P<0.01,{ }^{\star \star \star} P<0.001$. 
Table 3. Fatty acid (FA) composition of erythrocyte and plasma choline glycerophospholipids in children with Down syndrome (DS) when compared with their healthy siblings (HS)

(Mean values and standard deviations of total FA (\%), $n 11$ for HS and $n 9$ for DS)

\begin{tabular}{|c|c|c|c|c|c|c|c|c|}
\hline & \multicolumn{4}{|c|}{ Erythrocyte } & \multicolumn{4}{|c|}{ Plasma } \\
\hline & \multicolumn{2}{|c|}{$\mathrm{HS}$} & \multicolumn{2}{|c|}{ DS } & \multicolumn{2}{|c|}{$\mathrm{HS}$} & \multicolumn{2}{|c|}{ DS } \\
\hline & Mean & SD & Mean & SD & Mean & SD & Mean & SD \\
\hline $16: 0$ & $32 \cdot 88$ & $1 \cdot 10$ & $35 \cdot 56^{\star \star \star}$ & $2 \cdot 12$ & 27.66 & 2.47 & 28.73 & 1.43 \\
\hline $18: 0$ & 11.62 & 0.75 & $10 \cdot 65^{\star}$ & $1 \cdot 14$ & $13 \cdot 87$ & 1.06 & $12 \cdot 69^{*}$ & $1 \cdot 11$ \\
\hline$\Sigma$ SFA & $45 \cdot 12$ & 1.04 & $46.94^{\star}$ & 1.92 & $42 \cdot 34$ & $2 \cdot 30$ & $42 \cdot 19$ & 1.70 \\
\hline $16: 1 n-7$ & 0.49 & $0 \cdot 16$ & 0.48 & 0.17 & 0.65 & 0.12 & $0.93^{*}$ & 0.44 \\
\hline $18: 1 n-9$ & $16 \cdot 10$ & 0.97 & $16 \cdot 85$ & 1.43 & $11 \cdot 34$ & 1.72 & $12 \cdot 75$ & $3 \cdot 27$ \\
\hline $18: 1 n-7$ & 1.52 & 0.27 & $1.76^{*}$ & 0.16 & 1.34 & 0.25 & $1.66^{\star \star}$ & 0.16 \\
\hline ¿MUFA & 18.45 & 1.09 & $19 \cdot 37$ & 1.59 & $13 \cdot 62$ & 1.66 & $15 \cdot 60$ & $3 \cdot 71$ \\
\hline$\sum n-7$ & 2.02 & 0.39 & $2 \cdot 24$ & 0.21 & 1.99 & 0.30 & $2.59^{\star \star}$ & 0.47 \\
\hline $18: 0 / 18: 1$ & 0.66 & 0.07 & $0.58^{*}$ & 0.09 & $1 \cdot 11$ & 0.16 & $0.92^{*}$ & 0.23 \\
\hline $16: 0$ DMA & 0.62 & 0.09 & $0.51^{*}$ & 0.11 & 0.47 & 0.12 & 0.43 & 0.13 \\
\hline 18:0 DMA & 0.36 & $0 \cdot 12$ & $0.23^{\star}$ & 0.07 & 0.11 & 0.05 & $0 \cdot 10$ & 0.05 \\
\hline ¿DMA & 1.06 & $0 \cdot 16$ & $0.80^{* *}$ & 0.18 & 0.64 & 0.18 & 0.58 & 0.19 \\
\hline $18: 2 n-6$ & $21 \cdot 30$ & 1.64 & 19.91 & $2 \cdot 21$ & 24.84 & $2 \cdot 27$ & $23 \cdot 24$ & 3.07 \\
\hline $20: 4 n-6$ & $6 \cdot 49$ & 0.77 & $5 \cdot 65^{\star}$ & 0.73 & $8 \cdot 20$ & $1 \cdot 27$ & 8.00 & $1 \cdot 20$ \\
\hline $22: 4 n-6$ & 0.34 & 0.08 & 0.29 & 0.06 & 0.25 & 0.06 & 0.30 & 0.08 \\
\hline$\sum n-6$ PUFA & $31 \cdot 22$ & 1.83 & $28 \cdot 84^{\star \star}$ & 1.74 & $37 \cdot 24$ & $2 \cdot 22$ & 35.57 & $2 \cdot 79$ \\
\hline $18: 2 n-6 / 20: 4 n-6$ & 3.33 & 0.47 & $3 \cdot 61$ & 0.82 & $3 \cdot 13$ & 0.75 & 3.00 & 0.77 \\
\hline $22: 4 n-6 / 22: 5 n-6$ & $1 \cdot 17$ & 0.94 & 0.73 & 0.22 & 0.69 & 0.17 & $0.89^{*}$ & 0.19 \\
\hline $20: 5 n-3$ & 0.81 & 0.37 & 0.54 & 0.18 & 1.23 & 0.57 & 0.81 & 0.34 \\
\hline $22: 6 n-3$ & 1.87 & 0.69 & 1.81 & 0.74 & 3.08 & 1.27 & $3 \cdot 20$ & 1.23 \\
\hline$\sum n-3$ PUFA & 3.48 & 1.03 & $3 \cdot 22$ & 0.83 & 5.44 & 1.77 & 5.35 & 1.46 \\
\hline
\end{tabular}

DMA, fatty aldehyde dimethylacetals.

Mean value was significantly different from that of the HS group: ${ }^{*} P<0.05,{ }^{* *} P<0.01,{ }^{* \star *} P<0.001$.

Plasmalogen species are less abundant in ChoGpl when compared with PtdEtn. However, the amounts of 16:0 DMA and 18:0 DMA were significantly reduced in the erythrocyte membrane of children with DS, causing an approximately $24 \%$ decrease in total DMA. Changes in plasma ChoGpl plasmalogens were not statistically significant.

The amounts of total $n-6$ FA were reduced in the erythrocyte membrane ChoGpl of children with DS, mainly due to a $13 \%$ reduction in the amount of $20: 4 n-6$. The amount of $20: 5 n-3$ in the erythrocyte membrane and plasma ChoGpl of children with DS was statistically similar to that found for their healthy siblings; however, it showed a very strong tendency towards reduction $(P=0.060$ for erythrocytes and $P=0.067$ for plasma; Table 3).

The fourth PL fraction analysed in erythrocyte membrane samples was CerPCho (Table 4). Overall, a tendency of the increase in palmitic acid (16:0) values in the erythrocytes of children with DS was observed $(P=0 \cdot 067)$, but no significant differences in the total amounts of SFA were found. The total amount of MUFA was significantly increased, but no change in any individual MUFA reached statistical significance. The ratio 18:0/MUFA decreased, and this result is consistent in nearly all the fractions studied. The total amount of DMA tended to be lower in the erythrocyte membrane CerPCho of children with DS, but there was no increase in the amount of individual DMA FA, and this result was repetitive in nearly all the fractions studied. There were no statistically significant differences in the amount of $n-6$ or $n$-3 PUFA (Table 4).

CE represent a major lipid fraction in plasma. GC analysis revealed only minor changes in cholesterol esters SFA. As in erythrocyte membrane and plasma ChoGpl, the amount of 18:0 was decreased in children with DS. The amount of $18: 1 n-7$ was increased by $20 \%$ in plasma cholesterol samples of children with DS, but the values did not reach statistical significance as in other fractions. No differences were found for DMA or $n-6$ and $n-3$ PUFA. The amount of $20: 5 n-3$ in children with DS was not statistically different from that found in their healthy siblings, but similarly to erythrocyte and plasma ChoGpl data, it showed a strong tendency towards reduction $(P=0.064$; Table 4$)$. No differences were found in the FA composition of plasma TAG (data not shown).

\section{Antioxidant enzymes}

Enzyme activities of erythrocyte SOD, GPx and catalase were determined. Erythrocyte SOD activity $(\mathrm{U} / \mathrm{ml})$ was found to be increased by $25 \%$ in the blood samples of children with DS $(P=0.02$; Fig. 1). GPx activity ( $\mathrm{nmol} / \mathrm{min}$ per $\mathrm{ml}$ ) was increased by $40 \%$. No differences were found for catalase activities (data not shown).

\section{Phospholipid molecular species analysis}

PL molecular species in erythrocyte membrane were analysed by MS (ESI/quadrupole time-of-flight) analysis. This technique allows the resolution of intact PL in their native form directly from crude lipid extracts without prior chromatographic separation or derivatisation. Identification of all signals was carried out by the product-ion tandem MS/MS method based on characteristic fragmentation spectra for all peaks. Fig. 2(a) 
Table 4. Fatty acid (FA) composition of erythrocyte sphingomyelin (CerPCho) and plasma cholesterol esters (CE) in children with Down syndrome (DS) when compared with their healthy siblings (HS) (Mean values and standard deviations of total FA (\%), $n 11$ for HS and $n 9$ for DS)

\begin{tabular}{|c|c|c|c|c|c|c|c|c|}
\hline & \multicolumn{4}{|c|}{ CerPCho } & \multicolumn{4}{|c|}{ Plasma CE } \\
\hline & \multicolumn{2}{|c|}{ HS } & \multicolumn{2}{|c|}{ DS } & \multicolumn{2}{|c|}{ HS } & \multicolumn{2}{|c|}{ DS } \\
\hline & Mean & SD & Mean & SD & Mean & SD & Mean & SD \\
\hline $16: 0$ & 21.95 & 3.83 & $24 \cdot 74$ & 2.23 & $10 \cdot 36$ & 0.92 & $11 \cdot 18$ & $1 \cdot 12$ \\
\hline 18:0 & $14 \cdot 10$ & 3.92 & $12 \cdot 31$ & $2 \cdot 30$ & 0.94 & $0 \cdot 14$ & $0.76^{\star}$ & 0.18 \\
\hline$\Sigma$ SFA & 63.66 & 4.68 & 63.72 & 3.93 & $12 \cdot 18$ & $1 \cdot 13$ & $12 \cdot 78$ & 1.52 \\
\hline $16: 1 n-7$ & 0.15 & 0.13 & 0.11 & 0.06 & 2.55 & 0.85 & 2.33 & 0.98 \\
\hline $18: 1 n-9$ & $2 \cdot 48$ & 0.76 & $2 \cdot 71$ & 0.79 & 18.90 & 1.90 & $19 \cdot 73$ & 3.01 \\
\hline $18: 1 n-7$ & 0.28 & 0.07 & 0.32 & 0.09 & 0.96 & 0.24 & 1.15 & 0.19 \\
\hline ¿MUFA & 19.63 & 3.34 & $22 \cdot 33^{*}$ & 1.49 & 22.59 & $2 \cdot 13$ & 23.37 & $3 \cdot 70$ \\
\hline$\sum n-7$ & 0.43 & 0.15 & 0.42 & $0 \cdot 10$ & 3.51 & 1.00 & 3.49 & 1.06 \\
\hline $18: 0 / 18: 1$ & $5 \cdot 20$ & 0.88 & $4 \cdot 27^{*}$ & 1.08 & 0.05 & 0.01 & $0.04^{*}$ & 0.01 \\
\hline $18: 2 n-6$ & $2 \cdot 17$ & 0.80 & $2 \cdot 18$ & 0.53 & 53.90 & 3.05 & 53.00 & 4.98 \\
\hline $20: 4 n-6$ & $5 \cdot 10$ & 2.45 & $3 \cdot 61$ & 1.36 & $6 \cdot 22$ & 1.03 & $6 \cdot 27$ & 0.94 \\
\hline $22: 4 n-6$ & 1.09 & 0.70 & 0.79 & 0.41 & 0.03 & 0.02 & 0.03 & 0.02 \\
\hline$\Sigma n-6$ PUFA & 9.65 & $4 \cdot 21$ & $7 \cdot 71$ & 2.48 & $62 \cdot 27$ & 2.90 & $61 \cdot 18$ & 5.03 \\
\hline $18: 2 n-6 / 20: 4 n-6$ & 0.50 & 0.20 & 0.65 & 0.16 & 8.95 & 1.95 & 8.65 & 1.60 \\
\hline $22: 4 n-6 / 22: 5 n-6$ & 1.80 & 0.29 & 1.61 & 0.62 & 0.11 & 0.10 & 0.16 & 0.12 \\
\hline $20: 5 n-3$ & 0.19 & $0 \cdot 10$ & 0.18 & 0.15 & 1.15 & 0.65 & 0.71 & 0.22 \\
\hline $22: 6 n-3$ & 4.26 & 1.96 & 3.73 & 2.02 & 0.55 & 0.24 & 0.56 & 0.27 \\
\hline$\Sigma n-3$ PUFA & 5.95 & $2 \cdot 74$ & $5 \cdot 21$ & 2.23 & 2.36 & 0.90 & 1.97 & 0.50 \\
\hline
\end{tabular}

Mean value was significantly different from that of the HS group: ${ }^{\star} P<0.05,{ }^{\star \star} P<0.01,{ }^{* \star \star} P<0.001$.

shows a section of the positive-ion ESI-MS/MS spectrum of a crude lipid extract of erythrocyte membrane from children with DS. The major ChoGpl molecular species and the peak assignment are shown in Fig. 2(a).

Palmitic acid (16:0) is one of the major SFA in various erythrocyte membrane PL (5-35\% of total FA) and plasma ChoGpl and CerPCho (28 and $22 \%$ of total FA, respectively) (Tables 2-4). In the erythrocyte membrane ChoGpl of children with DS, significant changes in the distribution among 16:0-containing molecular species were found. ChoGpl species containing shorter-chain and less unsaturated FA in the $s n$ - 2 position (i.e. 16:0,18:1 and 18:2) were more than doubled (total increase from 16 to $40 \%$ of all choline phosphoacylglycerol species), while molecular species containing arachidonic acid (20:4n-6) were significantly reduced. A relevant reduction was observed in species containing DHA (22:6n-3), but it did not reach statistical significance. The last two species (16:0, 20:4n-6 and $16: 0,22: 5 n-3)$ combined corresponded to $21 \%$ of all species in healthy siblings, but only $15 \%$ in children with DS (Fig. 2(b)).

\section{Discussion}

A child with DS is at a higher risk of diabetes, obesity, CVD, dementia and early onset of $\mathrm{AD}$. Although lipids are considered to play a role in the aetiology of all these disorders, little is known as to whether or not there is a disturbance in membrane lipids in DS. The double expression of SOD in DS is thought to be a risk factor by promoting oxidative stress conditions. The brain is especially rich in highly unsaturated $\mathrm{FA}^{(31)}$, which are susceptible to peroxidation ${ }^{(14)}$. Therefore, the aim of the present study was to assess the status of membrane FA in children with DS compared with their unaffected siblings. There were no differences in age or sex among the children included in the study, and dietary analysis revealed that the average intakes of energy and nutrients were similar between siblings. Particular attention was paid to fat intake, and it was found to be statistically similar.

Chromosome 21 contains several genes that have been implicated in neurodegenerative mechanisms. These include $\mathrm{Cu} / \mathrm{ZnSOD}$ (SOD-1), E26 transformation-specific sequence (Ets-2) transcription factors, DS critical region 1 (DSCR1) stress-inducible factor, and the amyloid precursor protein $(A P P)$ genes. The accumulation of $\mathrm{A} \beta$ plaques is progressive across the lifespan in DS ${ }^{(32)}$. SOD-1 activity is found elevated

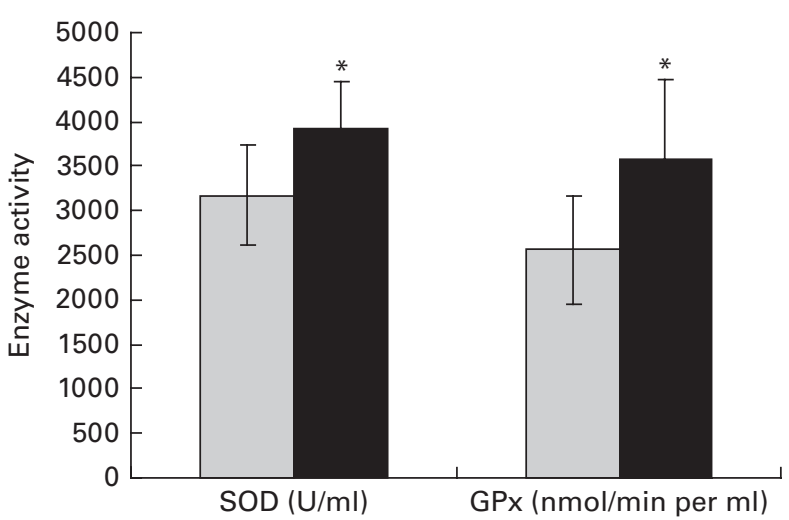

Fig. 1. Activities of erythrocyte superoxide dismutase (SOD: Down syndrome (DS, $n$ ), $n$ 8; healthy siblings (HS), $n$ 7) and glutathione peroxidase (GPx: DS, $n$ 9; control $(\square), n 7$ ) in children with DS when compared with their HS. Values are means, with their standard errors represented by vertical bars. ${ }^{*}$ Mean value was significantly different from that of the HS group $(P<0.05)$. One unit of SOD is defined as the amount of enzyme needed to exhibit $50 \%$ dismutation of the superoxide radical. 

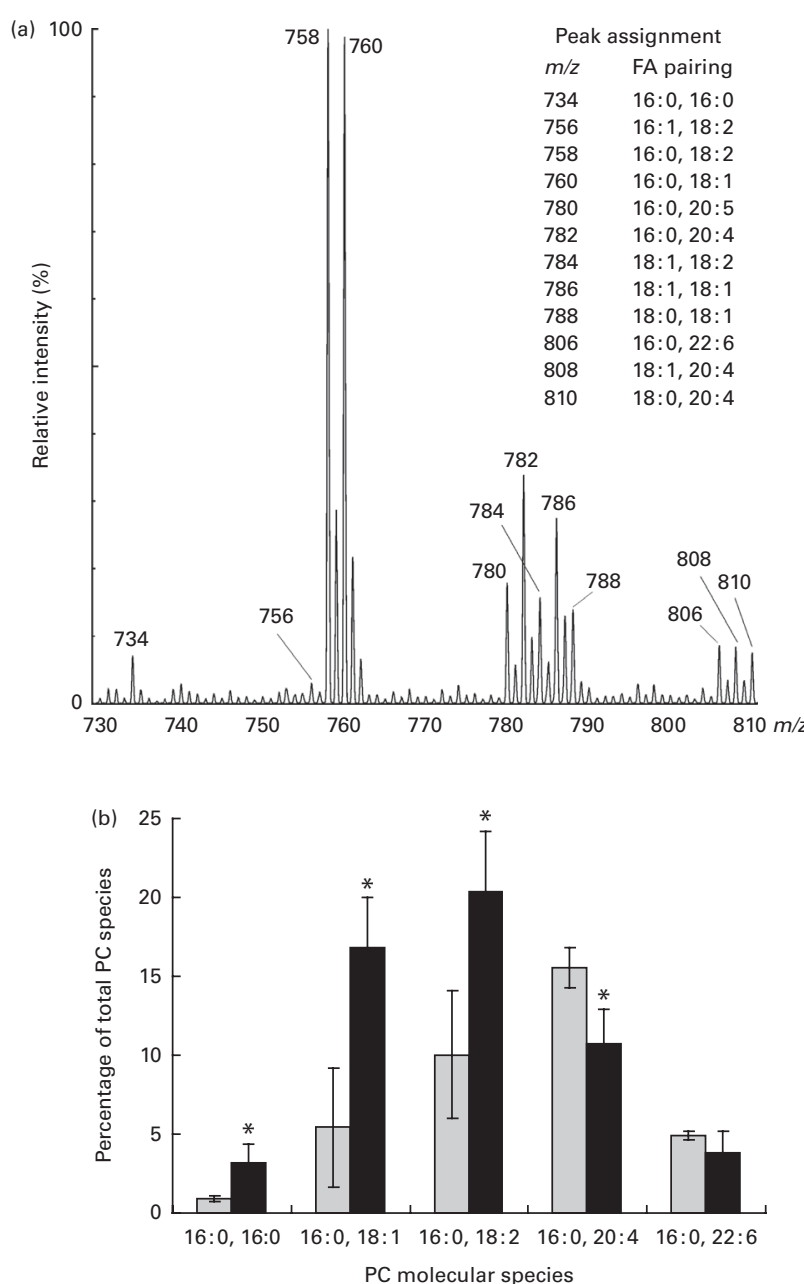

Fig. 2. Changes in choline phosphoacylglycerol (PC) molecular species in the erythrocyte membrane lipids of children with Down syndrome (DS, 口) when compared with their healthy siblings (HS). (a) Section of a representative positive-ion electrospray ionisation-MS/MS spectrum of erythrocyte membrane PC from a healthy sibling $\square$. The spectrum shows $[\mathrm{M}+\mathrm{H}]^{+}$ adducts only (see the 'Subjects and methods' section for details). (b) Palmitic acid (16:0)-containing PC species are expressed as the percentage of total erythrocyte membrane PC species. Values are means ( $\mathrm{HS}, n 5$; DS, $n 5)$, with standard deviations represented by vertical bars. * Mean value was significantly different from that of the HS group $(P<0.05) . m / z$, mass:charge ratio; FA, fatty acid.

in DS, which also occurs in conditions of oxidative stress, and is associated with a compensatory increase in GPx activity ${ }^{(33)}$. Enhanced free radical generation and anti-oxidative imbalance have been suggested to contribute to accelerated ageing and the prevalence of the neuropathology of $\mathrm{AD}$ observed in these individuals. In particular, erythrocyte GPx has been found to significantly correlate with deficits in memory function among adults with DS $^{(34)}$.

The present results demonstrate that already in young children, antioxidant enzyme activities of erythrocyte SOD and GPx were significantly elevated. Reynolds ${ }^{(35)}$ found that antioxidant supplementation did not improve the development of children with DS. Interestingly, a study assessing total serum antioxidant activity levels, but in contrast to previous reports, has found no significant difference between control individuals and those with DS ${ }^{(36)}$. However, increased serum concentrations of lipid peroxidation products, i.e. malondialdehyde, have been detected in blood samples from individuals with $\mathrm{DS}^{(7)}$, suggesting that enhanced oxidative stress conditions might affect membrane lipid composition and, in particular, the PUFA profile of PL.

Detailed analysis performed by using a combination of TLC and GC techniques revealed significant changes in the FA profile of PL fractions in erythrocyte membrane, as well as PL and neutral lipid fractions in plasma. The first specific finding was that shorter-chain (sixteen or eighteen carbons) and less unsaturated (none, one and two double bonds) FA (i.e. 16:0, 18:0, $18: 1$ and $18: 2 n$-6) were increased in most lipid fractions analysed, while longer-chain (twenty and twenty-two carbons) and highly unsaturated (four and five double bonds) FA (i.e. 20:4n-6 and 22:4n-6) were reduced. The amount of 20:5n-3 was not significantly reduced, but presented a strong tendency towards a reduction in erythrocyte membrane ChoGpl $(P=0.060)$, plasma ChoGpl $(P=0.067)$ and plasma cholesterol esters $(P=0.064)$. These perturbations might be a consequence of increased oxidative stress conditions in which highly unsaturated FA are oxidised and destroyed, and shorter-chain and less unsaturated dietary precursor FA are compensated for the loss in erythrocyte membrane or plasma lipids.

To corroborate the aforementioned suggestion, more evidence was found based on the changes in the erythrocyte plasmalogen content of children with DS. Plasmalogens consist of an aldehyde in the $s n-1$ position, giving rise to a single vinyl ether double bond and a long-chain hydrocarbon containing a carboxyl group esterified to the $s n-2$ position of the glycerol moiety possessing anywhere between two and six double bonds. It is the interplay between these two side chains that creates a powerful antioxidant system that resides in the hydrophobic domain to scavenge free radicals. Thus, plasmalogens have been proposed as natural antioxidants following oxidative stress insults based on their ability to scavenge reactive oxygen species, such as peroxyl radicals and singlet oxygen, as well as to chelate potentially harmful metal ions ${ }^{(37-39)}$. Furthermore, oxidation of the vinyl ether bond has been suggested to prevent the oxidation of adjacent PUFA, thus protecting PUFA from the consequences of free radical attacks and the resulting epoxide, being a relatively stable molecule $\mathrm{f}^{(40,41)}$. In the present study, erythrocyte membrane plasmalogen species detected mainly as 18:0 DMA were significantly reduced in the erythrocytes of children with DS, suggesting that chronic oxidative stress conditions may have targeted and destroyed these phosphoacylglycerols as a first line of defence. However, an increase in plasmalogen oxidation products can be harmful and may as well pose a problem in itself. Plasmalogen epoxides and $\alpha$-hydroxyaldehydes (powerful nucleophilic reagents that can attack other biomolecules) are known to accumulate in all chronic diseases such as atherosclerosis and myocardial infarction, upon ageing as well as in $\mathrm{AD}$ and other neuropathological conditions ${ }^{(42)}$. Plasmalogen deficiency has been described in other neurological disorders such as Zellweger syndrome $^{(43)}$ and cerebral adrenoleukodystrophy ${ }^{(44)}$. 
In addition to changes in the major FA profile, significant changes were detected for a minor FA that was found in all the fractions analysed. cis-Vaccenic acid (18:1n-7, 11-octadecenoic acid and asclepic acid) is an $n-7$ isomer of oleic acid (18:1n-9) and was elevated in the blood PL fractions of children with DS. While oleic acid is the major MUFA found in all cells and tissues, cis-vaccenic acid comprises only about $1 \%$ of all FA in the blood. However, incorporation of cis-vaccenic acid into the erythrocyte membrane has been shown to enhance the rate of adenylate cyclase activation through the P-receptors. Adenylate cyclase catalyses the conversion of ATP to $3^{\prime}, 5^{\prime}$-cyclic AMP, an important second-messenger molecule involved in signal transduction $^{(45)}$. The present results show a small, but highly significant $(P<0.005)$ increase in cis-vaccenic acid in the blood samples of children with DS, possibly implicating a potential disturbance of $3^{\prime}, 5^{\prime}$-cyclic AMP-related signalling cascades.

While cis-vaccenic acid is a minor FA in erythrocyte membrane, it is a major component of cardiolipin, a PL found exclusively in bacterial and mitochondrial membranes. It amounts up to $20 \%$ of the PL of the mitochondrial membrane, and its biological function in this organelle is clearly crucial $^{(46)}$. It is located mainly in the inner mitochondrial membrane, where it interacts with a large number of mitochondrial proteins. This interaction affects the functional activation of certain enzymes, especially those involved in oxidative phosphorylation. Cardiolipin is an important cofactor for cholesterol translocation from the outer to the inner mitochondrial membrane, which plays a specific role in the import of proteins into the mitochondria. It binds in a highly specific way to DNA in chromatin, and appears to have a functional role in the regulation of gene expression. As opposed to other PL, in cardiolipin, cis-vaccenic acid, not oleic acid, is the main octadecenoic acid present ${ }^{(47)}$.

In the present study, we analysed blood samples only and did not collect any data about possible changes in cis-vaccenic acid in mitochondrial membranes. However, our data advocate the importance to study the contents of cis-vaccenic acid in other tissues. The erythrocyte membrane is a plasma membrane with a similar composition to vascular endothelial cells, which have the highest plasma membrane:cytosol volume ratio. An abnormality in erythrocyte membrane is likely to reflect vascular abnormality ${ }^{(48)}$.

The lipid composition of cell membranes is directly influenced by environmental variables, particularly diet, as well as endogenous metabolic abnormalities (e.g. oxidative stress). It is likely that the changes found in the erythrocyte membrane of children with DS will also be found in the lipid profile of other tissues. Hence, the changes in cisvaccenic acid found in the blood samples of children with DS may reflect a similar increase in cis-vaccenic acid in mitochondrial membranes, which may have an effect on cellular energy metabolism, particularly in tissues with high energy consumption such as brain tissue.

In the present study, we used the ESI-MS/MS technique to study the molecular distribution of individual FA in their native form as intact PL, particularly because GC data revealed that ChoGpl of children with DS showed a highly significant increase in the amount of 16:0. We identified the pairing profile at the $s n-1$ and $s n-2$ positions within the intact PL molecules, a property that may underlie their mutual interaction with membrane proteins. ESI-MS/MS is an ionisation technique used for the mass spectrometric analysis of polar compounds that was initially developed by Fenn et $a{ }^{(49)}$. The utility of selective ESI ionisation is based on the differential propensity of each lipid class to acquire either positive or negative charges under the source high voltage, thus lipid classes can be separated through their endogenous electric potential. Each class of lipids can be resolved in the ionisation source (intra-source separation), and individual molecular species can be further identified by MS and/or tandem MS (quadrupole time-of-flight).

Our data reveal profound changes in the distribution of FA among the 16:0-containing molecular species. ChoGpl species containing shorter-chain (eighteen carbons) and less unsaturated (one and two double bonds) FA were almost doubled, while molecular species containing long-chain PUFA were reduced in children with DS. Of note is the contrast between children with DS and their healthy siblings in terms of the $16: 0,20: 4 n-6$ species of choline phosphoacylglycerols. In the unaffected siblings, the 16:0, 20:4n-6 species was greater than the same linoleic acid precursor species (16:0, 18:2n-6). However, in children with DS, the opposite was the case: $18: 2 n-6>20: 4 n-6$.

These changes are much more specific than the overall increase in the amount of 16:0 ChoGpl detected by GC analysis and, of course, not noticeable in the GC data. The study of molecular species provides additional information about the conventional GC analysis on the percentage of FA, as it is a description of lipids as they occur in the cell membrane. As cell membrane PL are in intimate and functional contact with executive proteins, this method is likely to be more biologically representative of tissue status and so potentially useful diagnostically. Variations in the molecular species profile may cause modification in the interaction between PL and the proteins anchored in them, possibly influencing cell-cell interactions and cell signalling cascades.

The specific difference observed in linoleic/arachidonic acid molecular species suggests a metabolic defect in the conversion of linoleic to arachidonic acid, and/or specific functional defects in the incorporation of these FA into membrane PL. It is plausible that alteration of a specific membrane PL is bringing about a different protein profile.

\section{Conclusion}

Lipid analysis of PL in plasma and erythrocyte membrane revealed significant changes in the FA profile of children with DS when compared with their healthy siblings, which are not related to the differences in their diet intake. Moreover, lipid abnormalities in erythrocyte membrane cannot be considered as an isolated phenomenon, but are likely to reflect abnormal changes in the lipid profile of other cells and tissues as well. Thus, establishing variations in the PL profile of other organs is of utmost interest. Increased levels of SFA coinciding 
with decreased amounts of PUFA denote drastic changes in the membrane lipid profile and may cause alterations in membrane physical properties and function. In addition, membrane PL PUFA serve as a molecular cache for NEFA, i.e. arachidonic acid and DHA and their metabolites (e.g. PG and neuroprotectins), which are key players in signalling pathways regulating cell metabolism and stress response. Taken together, alterations in membrane lipids found in the present study may help to explain the increased risk of developing CVD, metabolic and neurodegenerative diseases in children and adults with DS.

\section{Acknowledgements}

The present study is dedicated to the memory of Mr Peter Elliott, who was the founder of the Down Syndrome Research Foundation, for his commitment to Down Syndrome Research.

The authors thank the many families who participated in the study and to Dr R. Lister and Dr J. Brierley for their support and encouragement.

The present study was supported by the Down Syndrome Research Foundation (UK), the Mother and Child Foundation (UK) and the Letten Foundation (Norway). A. B. is grateful to Gulton Foundation (USA) for support.

The food diary analyses and all the experimental analyses related to the study were conducted at the Institute of Brain Chemistry and Human Nutrition, the laboratory which was at that time located at London Metropolitan University.

The authors' contributions are as follows: A. A. B., A. B., M. M. N., N. B. and M. A. C. designed the research, wrote the paper and had primary responsibility for the final content; C. L. assisted with the administration of the project; M. M. N. and N. B. recruited the families and analysed the food diaries; M. M. N., N. B. and M. A. C. conducted the interviews and liaison with parents and children; A. A. B. and A. B. conducted the research, analysed the data and performed the statistical analysis.

The authors have no conflict of interest to declare.

\section{References}

1. Devlin L \& Morrison PJ (2004) Accuracy of the clinical diagnosis of Down syndrome. Ulster Med J 73, 4-12.

2. Newberger DS (2000) Down syndrome: prenatal risk assessment and diagnosis. Am Fam Physician 62, 825-832, 837-838.

3. Harman D (2002) Alzheimer's disease: role of aging in pathogenesis. Ann N Y Acad Sci 959, 384-395 (discussion 463-465).

4. Zigman WB \& Lott IT (2007) Alzheimer's disease in Down syndrome: neurobiology and risk. Ment Retard Dev Disabil Res Rev 13, 237-246.

5. Mehta PD, Capone G, Jewell A, et al. (2007) Increased amyloid beta protein levels in children and adolescents with Down syndrome. J Neurol Sci 254, 22-27.

6. Schupf N, Patel B, Pang D, et al. (2007) Elevated plasma betaamyloid peptide Abeta(42) levels, incident dementia, and mortality in Down syndrome. Arch Neurol 64, 1007-1013.

7. Muchová J, Sustrová M, Garaiová I, et al. (2001) Influence of age on activities of antioxidant enzymes and lipid peroxidation products in erythrocytes and neutrophils of Down syndrome patients. Free Radic Biol Med 31, 499-508.

8. Ordonez FJ, Rosety-Plaza M \& Rosety-Rodriguez M (2006) Glucose-6-phosphatedehydrogenase is also increased in erythrocytes from adolescents with Down syndrome. Downs Syndr Res Pract 11, 84-87.

9. Schweber MS (1989) Alzheimer's disease and Down syndrome. Prog Clin Biol Res 317, 247-267.

10. Lott IT \& Head E (2001) Down syndrome and Alzheimer's disease: a link between development and aging. Ment Retard Dev Disabil Res Rev 7, 172-178.

11. Jovanovic SV, Clements D \& MacLeod K (1998) Biomarkers of oxidative stress are significantly elevated in Down syndrome. Free Radic Biol Med 25, 1044-1048.

12. Percy ME, Dalton AJ, Markovic VD, et al. (1990) Red cell superoxide dismutase, glutathione peroxidase and catalase in Down syndrome patients with and without manifestations of Alzheimer disease. Am J Med Genet 35, 459-467.

13. Pastor MC, Sierra C, Doladé M, et al. (1998) Antioxidant enzymes and fatty acid status in erythrocytes of Down's syndrome patients. Clin Chem 44, 924-929.

14. Yavin E, Brand A \& Green P (2002) Docosahexaenoic acid abundance in the brain: a biodevice to combat oxidative stress. Nutr Neurosci 5, 149-157.

15. Sinclair AJ \& Crawford MA (1972) Low-fat diets and the survival of newborn rats. Biochem J 126, 18P-19P.

16. Liu Q, Smith MA, Avilá J, et al. (2005) Alzheimer-specific epitopes of tau represent lipid peroxidation-induced conformations. Free Radic Biol Med 38, 746-754.

17. Bazan NG (2005) Neuroprotectin D1 (NPD1): a DHA-derived mediator that protects brain and retina against cell injury-induced oxidative stress. Brain Pathol 15, 159-166.

18. Lukiw WJ \& Bazan NG (2008) Docosahexaenoic acid and the aging brain. J Nutr 138, 2510-2514.

19. Samieri C, Féart C, Letenneur L, et al. (2008) Low plasma eicosapentaenoic acid and depressive symptomatology are independent predictors of dementia risk. Am J Clin Nutr 88, 714-721.

20. Palacios-Pelaez R, Lukiw WJ \& Bazan NG (2010) Omega-3 essential fatty acids modulate initiation and progression of neurodegenerative disease. Mol Neurobiol 41, 367-374.

21. Bakan E, Yildirim A, Kurtul N, et al. (2006) Effects of type 2 diabetes mellitus on plasma fatty acid composition and cholesterol content of erythrocyte and leukocyte membranes. Acta Diabetol 43, 109-113.

22. Younsi M, Quilliot D, Al-Makdissy N, et al. (2002) Erythrocyte membrane phospholipid composition is related to hyperinsulinemia in obese nondiabetic women: effects of weight loss. Metabolism 51, 1261-1268.

23. De Franceschi L, Olivieri O \& Corrocher R (2004) Erythrocyte aging in neurodegenerative disorders. Cell Mol Biol (Noisy-le-grand) 50, 179-185.

24. Thiel R \& Fowkes SW (2005) Can cognitive deterioration associated with Down syndrome be reduced? Med Hypotheses 64, 524-532.

25. McCance RA, Widdowson EM, Holland B, et al. (1991) Ministry of Agriculture, Royal Society of Chemistry, McCance and Widdowson's The Composition of Foods, 5th ed. Cambridge: Royal Society of Chemistry and Ministry of Agriculture, Fisheries and Food.

26. Wynn SW, Wynn AH, Doyle W, et al. (1994) The association of maternal social class with maternal diet and the dimensions of babies in a population of London women. Nutr Health 9 , 303-315.

27. Folch J, Lees M \& Sloane Stanley GH (1957) A simple method for the isolation and purification of total lipides from animal tissues. J Biol Chem 226, 497-509. 
28. Bueno AA, Ghebremeskel K, Bakheit KH, et al. (2012) Dimethyl acetals, an indirect marker of the endogenous antioxidant plasmalogen level, are reduced in blood lipids of Sudanese pre-eclamptic subjects whose background diet is high in carbohydrate. J Obstet Gynaecol 32, 241-246.

29. Brand A, Crawford MA \& Yavin E (2010) Retailoring docosahexaenoic acid-containing phospholipid species during impaired neurogenesis following omega-3 alpha-linolenic acid deprivation. J Neurochem 114, 1393-1404.

30. Han X \& Gross RW (1995) Structural determination of picomole amounts of phospholipids via electrospray ionization tandem mass spectrometry. J Am Soc Mass Spectrom 6, 1202-1210.

31. Crawford MA \& Sinclair AJ (1972) Nutritional influences in the evolution of the mammalian brain. In Lipids, Malnutrition and the Developing Brain, pp. 267-292 [K Elliot and J Knight, editors]. Amsterdam: Elsevier (A Ciba Foundation Symposium).

32. Lott IT, Head E, Doran E, et al. (2006) Beta-amyloid, oxidative stress and Down syndrome. Curr Alzheimer Res 3, 521-528.

33. Meguid NA, Kholoussi NM \& Afifi HH (2001) Evaluation of superoxide dismutase and glutathione peroxidase enzymes and their cofactors in Egyptian children with Down's syndrome. Biol Trace Elem Res 81, 21-28.

34. Brugge K, Nichols S, Saitoh T, et al. (1999) Correlations of glutathione peroxidase activity with memory impairment in adults with Down syndrome. Biol Psychiatry 46, 1682-1689.

35. Reynolds $\mathrm{T}$ (2008) Antioxidants do not improve early childhood development in children with Down's syndrome. J Pediatr 153, 441.

36. Varga P, V Oláh A \& Oláh E (2008) Biochemical alterations in patients with Down syndrome. Orv Hetil 149, 1203-1213.

37. Zoeller RA, Lake AC, Nagan N, et al. (1999) Plasmalogens as endogenous antioxidants: somatic cell mutants reveal the importance of the vinyl ether. Biochem J 338, 769-776.

38. Leray C, Cazenave JP \& Gachet C (2002) Platelet phospholipids are differentially protected against oxidative degradation by plasmalogens. Lipids 37, 285-290.
39. Spiteller G (2006) Peroxyl radicals: inductors of neurodegenerative and other inflammatory diseases. Their origin and how they transform cholesterol, phospholipids, plasmalogens, polyunsaturated fatty acids, sugars, and proteins into deleterious products. Free Radic Biol Med 41, 362-387.

40. Zommara M, Tachibana N, Mitsui K, et al. (1995) Inhibitory effect of ethanolamine plasmalogen on iron- and copper-dependent lipid peroxidation. Free Radic Biol Med 18, 599-602.

41. Engelmann B (2004) Plasmalogens: targets for oxidants and major lipophilic antioxidants. Biochem Soc Trans 32, $147-150$

42. Lessig J \& Fuchs B (2009) Plasmalogens in biological systems: their role in oxidative processes in biological membranes, their contribution to pathological processes and aging and plasmalogen analysis. Curr Med Chem 16, 2021-2041.

43. Saitoh M, Itoh M, Takashima S, et al. (2009) Phosphatidyl ethanolamine with increased polyunsaturated fatty acids in compensation for plasmalogen defect in the Zellweger syndrome brain. Neurosci Lett 449, 164-167.

44. Khan M, Singh J \& Singh I (2008) Plasmalogen deficiency in cerebral adrenoleukodystrophy and its modulation by lovastatin. J Neurochem 106, 1766-1779.

45. Henis YI, Rimon G \& Felder S (1982) Lateral mobility of phospholipids in turkey erythrocytes. Implications for adenylate cyclase activation. J Biol Chem 257, 1407-1411.

46. Schlame M, Brody S \& Hostetler KY (1993) Mitochondrial cardiolipin in diverse eukaryotes. Comparison of biosynthetic reactions and molecular acyl species. Eur $J$ Biochem 212, 727-735.

47. Wolff RL, Combe NA \& Entressangles B (1985) Positional distribution of fatty acids in cardiolipin of mitochondria from 21-day-old rats. Lipids 20, 908-914.

48. Crawford MA, Golfetto I, Ghebremeskel K, et al. (2003) The potential role for arachidonic and docosahexaenoic acids in protection against some central nervous system injuries in preterm infants. Lipids 38, 303-315.

49. Fenn JB, Mann M, Meng CK, et al. (1989) Electrospray ionization for mass spectrometry of large biomolecules. Science 246, 64-71. 